PROCEEDINGS OF THE

AMERICAN MATHEMATICAL SOCIETY

Volume 125, Number 4, April 1997, Pages 1055-1062

S 0002-9939(97)03740-4

\title{
ON THE VON NEUMANN-JORDAN CONSTANT FOR BANACH SPACES
}

\author{
MIKIO KATO AND YASUJI TAKAHASHI
}

(Communicated by Palle E. T. Jorgensen)

\begin{abstract}
Let $C_{\mathrm{NJ}}(E)$ be the von Neumann-Jordan constant for a Banach space $E$. It is known that $1 \leq C_{\mathrm{NJ}}(E) \leq 2$ for any Banach space $E$; and $E$ is a Hilbert space if and only if $C_{\mathrm{NJ}}(E)=1$. We show that: (i) If $E$ is uniformly convex, $C_{\mathrm{NJ}}(E)$ is less than two; and conversely the condition $C_{\mathrm{NJ}}(E)<2$ implies that $E$ admits an equivalent uniformly convex norm. Hence, denoting by $\widetilde{C}_{\mathrm{NJ}}(E)$ the infimum of all von Neumann-Jordan constants for equivalent norms of $E, E$ is super-reflexive if and only if $\widetilde{C}_{\mathrm{NJ}}(E)<2$. (ii) If $\widetilde{C}_{\mathrm{NJ}}(E)=2^{2 / p-1}, 1<p \leq 2$ (the same value as that of $L_{p}$-space), $E$ is of Rademacher type $r$ and cotype $r^{\prime}$ for any $r$ with $1 \leq r<p$, where $1 / r+1 / r^{\prime}=1$; the converse holds if $E$ is a Banach lattice and $l_{p}$ is finitely representable in $E$ or $E^{\prime}$.
\end{abstract}

\section{INTRODUCTION AND PRELIMINARIES}

In connection with the famous work [8] of Jordan and von Neumann concerning inner products, the von Neumann-Jordan (NJ-) constant $C_{\mathrm{NJ}}(E)$ for a Banach space $E$ was introduced by Clarkson [3] as the smallest constant $C$ for which

$$
\frac{1}{C} \leq \frac{\|x+y\|^{2}+\|x-y\|^{2}}{2\left(\|x\|^{2}+\|y\|^{2}\right)} \leq C
$$

holds for all $x, y \in E$ with $(x, y) \neq(0,0)$. As is easily seen, if $C$ is best possible in the right-hand side inequality of (1), then so is $1 / C$ in the left. Despite its fundamental nature very little is known about the NJ-constant; a summary is given in Theorem A below. In what follows, let $1 \leq p, q, r, t \leq \infty$ unless otherwise stated, and let $p^{\prime}, q^{\prime}, r^{\prime}, t^{\prime}$ be their conjugate numbers, respectively.

A. Theorem. (i) $1 \leq C_{\mathrm{NJ}}(E) \leq 2$ for all Banach spaces $E$; and $C_{\mathrm{NJ}}(E)=1$ if and only if $E$ is a Hilbert space (Jordan and von Neumann [8]).

(ii) $C_{\mathrm{NJ}}\left(L_{p}\right)=2^{2 / t-1}$, where $t=\min \left\{p, p^{\prime}\right\}$ (Clarkson [3]; see also [5]).

(iii) For $L_{p}\left(L_{q}\right), L_{q}$-valued $L_{p}$-space (on arbitrary measure spaces), $C_{\mathrm{NJ}}\left(L_{p}\left(L_{q}\right)\right.$ ) $=2^{2 / t-1}$, where $t=\min \left\{p, q, p^{\prime}, q^{\prime}\right\} ;$ and for the Sobolev space $W_{p}^{k}(\Omega), C_{\mathrm{NJ}}\left(W_{p}^{k}(\Omega)\right)$ $=2^{2 / t-1}$, where $t=\min \left\{p, p^{\prime}\right\}$ (Kato and Miyazaki $\left.[10]\right)$.

Received by the editors September 8, 1995.

1991 Mathematics Subject Classification. Primary 46B20, 46B03, 46B42.

Key words and phrases. von Neumann-Jordan constant, uniform convexity, super-reflexivity, type and cotype, finite representability, $p$-convexity and $p$-concavity for a Banach lattice.

The authors were supported in part by Grants-in-Aid for Scientific Research from the Ministry of Education, Science and Culture (07640225 (first author), 07640240 (second author)). 
(iv) For $E=C_{c}(K)$, resp. $C_{b}(K)$ (the spaces of continuous functions on a locally compact Hausdorff space $K$ which have compact support, resp. are bounded), $C_{\mathrm{NJ}}(E)=2$ (Kato and Miyazaki $\left.[9]\right)$.

The following facts are readily seen:

B. Proposition. (i) $C_{\mathrm{NJ}}(E)=2^{2 / t-1}, 1 \leq t \leq 2$, if and only if

$$
\left\|A: l_{2}^{2}(E) \rightarrow l_{2}^{2}(E)\right\|=2^{1 / t}
$$

where $A=\left(\begin{array}{rr}1 & 1 \\ 1 & -1\end{array}\right)$ and $l_{2}^{2}(E)$ denotes the E-valued $l_{2}^{2}$-space; and hence

(ii) $C_{\mathrm{NJ}}\left(E^{\prime}\right)=C_{\mathrm{NJ}}(E)$, where $E^{\prime}$ is the dual space of $E$. (This was observed for $L_{p}$ in Clarkson [3].)

In this paper we first show that a uniformly convex Banach space $E$ is nearly characterized by the condition $C_{\mathrm{NJ}}(E)<2$; more precisely the uniform convexity for $E$ implies $C_{\mathrm{NJ}}(E)<2$, while conversely $C_{\mathrm{NJ}}(E)<2$ assures the existence of an equivalent uniformly convex norm in $E$. Hence, letting $\widetilde{C}_{\mathrm{NJ}}(E)$ be the infimum of all NJ-constants for equivalent norms of $E, E$ is super-reflexive if and only if $\widetilde{C}_{\mathrm{NJ}}(E)<2$. Secondly we show that if $\widetilde{C}_{\mathrm{NJ}}(E)=2^{2 / p-1}, 1<p \leq 2$ (the same value as that of $L_{p}$ and of $L_{p^{\prime}}$ as well), then $E$ is of type $r$ and cotype $r^{\prime}$ for any $r$ with $1 \leq r<p$; and the converse holds if $E$ is a Banach lattice and $l_{p}$ is finitely representable in $E$ or $E^{\prime}$. Thus, for a Banach lattice $E$ the number $p$ satisfying $\widetilde{C}_{\mathrm{NJ}}(E)=2^{2 / p-1}$ is coincident with $\min \left\{p(E), q(E)^{\prime}\right\}$, where $p(E)=$ $\sup \{p ; E$ is of type $p\}, q(E)=\inf \{q ; E$ is of cotype $q\}$ and $1 / q(E)+1 / q(E)^{\prime}=1$.

Let us recall some definitions. A Banach space $E$ is called strictly convex if $\|(x+y) / 2\|<1$ whenever $\|x\|=\|y\|=1, x \neq y$. E is said to be uniformly convex provided for each $\varepsilon(0<\varepsilon<2)$ there exists a $\delta>0$ such that $\|(x+y) / 2\|<$ $1-\delta$ whenever $\|x-y\| \geq \varepsilon,\|x\|=\|y\|=1$. $E$ is called $(2, \varepsilon)$-convex, $\varepsilon>0$, if $\min \{\|x+y\|,\|x-y\|\} \leq 2(1-\varepsilon)$ whenever $\|x\|=\|y\|=1$.

A Banach space $F$ is said to be finitely representable in $E$ provided for any $\lambda>1$ and each finite-dimensional subspace $F_{1}$ of $F$, there is an isomorphism $T$ of $F_{1}$ into $E$ for which

$$
\lambda^{-1}\|x\| \leq\|T x\| \leq \lambda\|x\| \text { for all } x \in F_{1} .
$$

$E$ is said to be super-reflexive ([7]; cf. [1], [12]) if no non-reflexive Banach space is finitely representable in $E$.

Super-reflexive spaces are characterized as those uniformly convexifiable:

C. Theorem (Enflo [4]; cf. [1], [15]). A Banach space E is super-reflexive if and only if $E$ admits an equivalent uniformly convex norm.

A Banach space $E$ is said to be of (Rademacher) type $p(1 \leq p \leq 2)$, resp. of cotype $q(2 \leq q \leq \infty)$, if there exists some $M>0$ such that

$$
\int_{0}^{1}\left\|\sum_{j=1}^{n} r_{j}(t) x_{j}\right\| d t \leq M\left\{\sum_{j=1}^{n}\left\|x_{j}\right\|^{p}\right\}^{1 / p},
$$

resp.

$$
\left\{\sum_{j=1}^{n}\left\|x_{j}\right\|^{q}\right\}^{1 / q} \leq M \int_{0}^{1}\left\|\sum_{j=1}^{n} r_{j}(t) x_{j}\right\| d t
$$


for all finite systems $\left\{x_{j}\right\}$ in $E$, where $r_{j}(t)$ are the Rademacher functions, i.e., $r_{j}(t)=\operatorname{sgn}\left(\sin 2^{j} \pi t\right)$.

D. Theorem (Maurey and Pisier [14]; also [16], esp. Theorem 3.11, or [12],v. 3). Let $E$ be a Banach space of infinite dimension. Let

$$
p(E):=\sup \{p ; E \text { is of type } p\},
$$

resp.

$$
q(E):=\inf \{q ; E \text { is of cotype } q\}
$$

Then

$$
p(E)=\min \left\{p ; l_{p} \text { is finitely representable in } E\right\},
$$

resp.

$$
q(E)=\max \left\{q ; l_{q} \text { is finitely representable in } E\right\} .
$$

(Note that $p(E) \leq 2 \leq q(E)$.)

A Banach lattice $E$ is called $p$-convex, resp. p-concave, $1 \leq p<\infty$, if there exists a constant $M$ such that

$$
\left\|\left\{\sum_{j=1}^{n}\left|x_{j}\right|^{p}\right\}^{1 / p}\right\| \leq M\left\{\sum_{j=1}^{n}\left\|x_{j}\right\|^{p}\right\}^{1 / p},
$$

resp.

$$
\left\{\sum_{j=1}^{n}\left\|x_{j}\right\|^{p}\right\}^{1 / p} \leq M\left\|\left\{\sum_{j=1}^{n}\left|x_{j}\right|^{p}\right\}^{1 / p}\right\|,
$$

for all finite systems $\left\{x_{j}\right\}$ in $E$. The smallest possible value of $M$ in (2), resp. (3), is called the p-convexity, resp. p-concavity, constant for $E$.

Now, we begin with the following proposition which will give effective examples later.

1. Proposition. Let $1<p \leq 2$ and $\lambda>1$. Let $X_{p, \lambda}$ be the space $l_{p^{\prime}}$ equipped with the norm $\|x\|_{p, \lambda}:=\max \left\{\|x\|_{p^{\prime}}, \lambda\|x\|_{\infty}\right\}$, where $1 / p+1 / p^{\prime}=1$. Then:

(i) $C_{\mathrm{NJ}}\left(X_{p, \lambda}\right)=\left\{\begin{array}{ll}\lambda^{2} 2^{2 / p-1} & \text { if } 1<\lambda \leq 2^{1 / p^{\prime}} \\ 2 & \text { if } \lambda \geq 2^{1 / p^{\prime}},\end{array}\right.$,

(ii) $X_{2, \lambda}$ is isomorphic to a Hilbert space and

$$
C_{\mathrm{NJ}}\left(X_{2, \lambda}\right)=\min \left\{\lambda^{2}, 2\right\},
$$

(iii) $X_{p, \lambda}$ is not strictly convex for any $\lambda>1$.

Proof. (i) Since

$$
\|x\|_{p^{\prime}} \leq\|x\|_{p, \lambda} \leq \lambda\|x\|_{p^{\prime}} \quad \text { for all } x \in X_{p, \lambda},
$$

we have

$$
\begin{aligned}
\|x+y\|_{p, \lambda}^{2}+\|x-y\|_{p, \lambda}^{2} & \leq \lambda^{2}\left(\|x+y\|_{p^{\prime}}^{2}+\|x-y\|_{p^{\prime}}^{2}\right) \\
& \leq \lambda^{2} 2^{2 / p}\left(\|x\|_{p^{\prime}}^{2}+\|y\|_{p^{\prime}}^{2}\right) \\
& \leq \lambda^{2} 2^{2 / p}\left(\|x\|_{p, \lambda}^{2}+\|y\|_{p, \lambda}^{2}\right),
\end{aligned}
$$


where the second inequality follows from the fact $C_{\mathrm{NJ}}\left(l_{p^{\prime}}\right)=2^{2 / p-1}$ (Theorem A (ii)) and Proposition B (i). This implies $C_{\mathrm{NJ}}\left(X_{p, \lambda}\right) \leq \lambda^{2} 2^{2 / p-1}$. Let $1<\lambda \leq 2^{1 / p^{\prime}}$. Put $x=(1 / \lambda, 1 / \lambda, 0, \ldots), y=(1 / \lambda,-1 / \lambda, 0, \ldots) \in X_{p, \lambda}$. Then, since $\|x\|_{p^{\prime}}=$ $\|y\|_{p^{\prime}}=\lambda^{-1} 2^{1 / p^{\prime}} \geq 1$ and $\|x\|_{\infty}=\|y\|_{\infty}=\lambda^{-1}$, we have

$$
\|x\|_{p, \lambda}=\|y\|_{p, \lambda}=\lambda^{-1} 2^{1 / p^{\prime}}
$$

and clearly

$$
\|x+y\|_{p, \lambda}=\|x-y\|_{p, \lambda}=2 .
$$

Hence,

$$
\frac{\|x+y\|_{p, \lambda}^{2}+\|x-y\|_{p, \lambda}^{2}}{2\left(\|x\|_{p, \lambda}^{2}+\|y\|_{p, \lambda}^{2}\right)}=\lambda^{2} 2^{2 / p-1}
$$

which implies $C_{\mathrm{NJ}}\left(X_{p, \lambda}\right)=\lambda^{2} 2^{2 / p-1}$. Let $\lambda \geq 2^{1 / p^{\prime}}$, and let $x$ and $y$ be as above. Then we have $\|x\|_{p, \lambda}=\|y\|_{p, \lambda}=1$ and (5). Therefore the left side of (6), in this case, takes the value two, or $C_{\mathrm{NJ}}\left(X_{p, \lambda}\right)=2$.

(ii) is clear by (4) and (i).

(iii) Take $\alpha$ as $(1 / \lambda)^{p^{\prime}}+\alpha^{p^{\prime}} \leq 1$ and $0<\alpha \leq 1 / \lambda$. Put $x=(1 / \lambda, 0,0, \ldots)$ and $y=(1 / \lambda, \alpha, 0, \ldots)$. Then $\|x\|_{p, \lambda}=\|y\|_{p, \lambda}=1$, whereas $\|x+y\|_{p, \lambda}=2$, so $X$ is not strictly convex. This completes the proof.

2. Theorem. Let $E$ be uniformly convex. Then $C_{\mathrm{NJ}}(E)<2$.

Proof. Let $E$ be uniformly convex. Let $\varepsilon$ be any positive number with $0<\varepsilon<2^{1 / 2}$. Then there exists a $\delta>0$ such that $\|x\| \leq 1,\|y\| \leq 1$, and $\|x-y\| \geq \varepsilon$ imply $\|(x+y) / 2\|^{2} \leq(1-\delta)\left[\left(\|x\|^{2}+\|y\|^{2}\right) / 2\right]$ (see [1], p. 190, or [11], p. 360). Let $x$ and $y$ be any elements in $E$ with $\|x\|^{2}+\|y\|^{2}=1$. We first assume that $\|x-y\| \geq \varepsilon$. Then

$$
\left\|\frac{x+y}{2}\right\|^{2}+\left\|\frac{x-y}{2}\right\|^{2} \leq(1-\delta) \frac{\|x\|^{2}+\|y\|^{2}}{2}+\frac{\|x\|^{2}+\|y\|^{2}}{2} \leq 1-\frac{\delta}{2},
$$

and hence

$$
\|x+y\|^{2}+\|x-y\|^{2} \leq 2(2-\delta) .
$$

Next, let $\|x-y\| \leq \varepsilon$. Then

$$
\|x+y\|^{2}+\|x-y\|^{2} \leq 2\left(\|x\|^{2}+\|y\|^{2}\right)+\varepsilon^{2} \leq 2\left(1+\varepsilon^{2} / 2\right) .
$$

Consequently, by (7) and (8) we have

$$
\frac{\|x+y\|^{2}+\|x-y\|^{2}}{2\left(\|x\|^{2}+\|y\|^{2}\right)} \leq 1+\max \left\{1-\delta, \varepsilon^{2} / 2\right\},
$$

or $C_{\mathrm{NJ}}(E)<2$.

The converse of Theorem 2 is not true:

3. Theorem. For any $\varepsilon>0$ there exists a Banach space $E$, isomorphic to a Hilbert space, with $C_{\mathrm{NJ}}(E)<1+\varepsilon$ which is not strictly convex.

In fact, for the spaces $X_{2, \lambda}(\lambda>1)$, we have $C_{\mathrm{NJ}}\left(X_{2, \lambda}\right) \rightarrow 1$ as $\lambda \rightarrow 1$, whereas $X_{2, \lambda}$ is not strictly convex.

Although the condition $C_{\mathrm{NJ}}(E)<2$ does not even imply strict convexity for $E$, it assures the existence of an equivalent norm on $E$ for which $E$ becomes uniformly convex (cf. Theorem C). 
4. Theorem. Let $C_{\mathrm{NJ}}(E)<2$. Then $E$ is super-reflexive; the converse is not true.

Proof. Assume $C:=C_{\mathrm{NJ}}(E)<2$. We see that $E$ is $(2, \varepsilon)$-convex with some $\varepsilon$, which implies the super-reflexivity for $E$ (James [6]; see also [1], [12]). Let $x$ and $y$ be any elements in $E$ with $\|x\|=\|y\|=1$. Then

$$
\begin{aligned}
\min _{\varepsilon_{i}= \pm 1}\left\|\varepsilon_{1} x+\varepsilon_{2} y\right\| & \leq\left\{\frac{1}{2}\left(\|x+y\|^{2}+\|x-y\|^{2}\right)\right\}^{1 / 2} \\
& \leq C^{1 / 2}\left(\|x\|^{2}+\|y\|^{2}\right)^{1 / 2} \\
& =(2 C)^{1 / 2}=2(1-\varepsilon),
\end{aligned}
$$

where $\varepsilon=1-(C / 2)^{1 / 2}$. For the latter assertion consider the space $X_{2, \sqrt{2}}$. Indeed, $X_{2, \sqrt{2}}$ is isomorphic to a Hilbert space and hence super-reflexive, whereas $C_{\mathrm{NJ}}\left(X_{2, \sqrt{2}}\right)=2$ by Proposition 1. ( $l_{1}^{n}$ and $l_{\infty}^{n}$ are also such examples.)

5. Definition. Let $\widetilde{C}_{\mathrm{NJ}}(E)$ denote the infimum of all von Neumann-Jordan constants for equivalent norms of a Banach space $E$.

Owing to Theorems 2 and 4, we obtain the following theorem.

6. Theorem. A Banach space $E$ is super-reflexive if and only if $\widetilde{C}_{\mathrm{NJ}}(E)<2$.

So far we have treated Banach spaces whose NJ-constant is less than two or can be so taken by renorming. Let us now consider the spaces with NJ-constant $2^{2 / t-1}$, $1<t \leq 2$, which is the same as that of $L_{p}$-space if $t=\min \left\{p, p^{\prime}\right\}$. We first note the following facts; the proof is easy and is omitted.

7. Proposition. (i) Let $F$ be a Banach space which is finitely representable in E. Then $C_{\mathrm{NJ}}(F) \leq C_{\mathrm{NJ}}(E)$.

(ii) If $l_{p}$ is finitely representable in $E, C_{\mathrm{NJ}}(E) \geq 2^{2 / t-1}$, where $t=\min \left\{p, p^{\prime}\right\}$.

8. Proposition. Let $C_{\mathrm{NJ}}(E) \leq 2^{2 / p-1}, 1<p \leq 2$. Then $E$ is of type $r$ and cotype $r^{\prime}$ for any $r$ with $1 \leq r<p$. The converse is not true.

Proof. Assume $C_{\mathrm{NJ}}(E) \leq 2^{2 / p-1}$. Suppose that $E$ is not of type $r$ for some $r<p$. Then $l_{r}$ is finitely representable in $E$ by Theorem D. Hence, $C_{\mathrm{NJ}}(E) \geq 2^{2 / r-1}>$ $2^{2 / p-1}$ by the preceding proposition, which is a contradiction. By Proposition B, $E^{\prime}$ is also of type $r$ for any $r<p$, which implies that $E$ is of cotype $r^{\prime}$ for any such $r$ (cf. [13], Proposition 1.e.17; or [1], p. 309).

The latter part is a consequence of a result by Pisier and $\mathrm{Xu}$ [17] which asserts that for any $q>2$ there exists a non-reflexive Banach space $E\left(C_{\mathrm{NJ}}(E)=2\right)$ such that $E$ is of type 2 and cotype $q$.

9. Remark. Propositions 7 and 8 remain valid if $C_{\mathrm{NJ}}(E)$ is replaced by $\widetilde{C}_{\mathrm{NJ}}(E)$ (note that "finite representability" and "type, cotype" are isomorphic properties).

For a Banach lattice the situation in Proposition 8 or Remark 9 is preferable, and we obtain the following result.

10. Theorem. Let $E$ be a Banach lattice and let $1<p \leq 2$. Then the following assertions are equivalent:

(i) $\widetilde{C}_{\mathrm{NJ}}(E) \leq 2^{2 / p-1}$,

(ii) $E$ is of type $r$ and cotype $r^{\prime}$ for any $r<p$, 
(iii) $E$ and $E^{\prime}$ are of type $r$ for any $r<p$,

(iv) $E$ and $E^{\prime}$ are of cotype $r^{\prime}$ for any $r<p$,

(v) $E$ is $r$-convex and $r^{\prime}$-concave for any $r<p$.

For the proof of Theorem 10 we need the following two lemmas.

11. Lemma ([13], Corollary 1.f.9). Let $1<r<\infty$. Let $E$ be a Banach lattice of type $r$, resp. cotype $r$. Then $E$ is $(r-\varepsilon)$-convex, resp. $(r+\varepsilon)$-concave, for any $\varepsilon>0$.

The following lemma is an easy consequence of Theorem 1.d.1 of [13] (see also the proof of Theorem 1.f.1 of [13]).

12. Lemma (cf. [13], Theorem 1.d.1). Let $1 \leq p \leq 2$. Let E be a Banach lattice. Then, for any $x$ and $y$ in $E$,

$$
\left(|x+y|^{p^{\prime}}+|x-y|^{p^{\prime}}\right)^{1 / p^{\prime}} \leq 2^{1 / p^{\prime}}\left(|x|^{p}+|y|^{p}\right)^{1 / p}
$$

Proof of Theorem 10. We have already seen that the implications (i) $\Rightarrow$ (iii) $\Rightarrow$ (ii) are valid in the proof of Proposition 8 and Remark 9. The implication (ii) $\Rightarrow$ (iv) is clear, since if $E$ is of type $r$, then $E^{\prime}$ is of cotype $r^{\prime}$ (cf. [13], Proposition 1.e.17; or [1], p. 309).

(iv) $\Rightarrow(\mathrm{v})$ : Assume that $E$ and $E^{\prime}$ are of cotype $r^{\prime}$ for any $r<p$. Then by Lemma $11, E$ and $E^{\prime}$ are $r^{\prime}$-concave for any $r<p$, and the latter implies $r$-convexity of $E$ (see [13], Proposition 1.d.4).

$(\mathrm{v}) \Rightarrow(\mathrm{i})$ : Let $E$ be $r$-convex and $r^{\prime}$-concave for any $r<p$. Here we can take both $r$-convex and $r^{\prime}$-concave constants to be one by a suitable renorming ([13], Proposition 1.d.8). We denote such an equivalent norm by $\|\cdot\|_{1}$. Then by Lemma 12 we have

$$
\begin{aligned}
\left(\|x+y\|_{1}^{2}+\|x-y\|_{1}^{2}\right)^{1 / 2} & \leq 2^{1 / 2-1 / r^{\prime}}\left(\|x+y\|_{1}^{r^{\prime}}+\|x-y\|_{1}^{r^{\prime}}\right)^{1 / r^{\prime}} \\
& \leq 2^{1 / 2-1 / r^{\prime}}\left\|\left(|x+y|^{r^{\prime}}+|x-y|^{r^{\prime}}\right)^{1 / r^{\prime}}\right\|_{1} \\
& \leq 2^{1 / 2}\left\|\left(|x|^{r}+|y|^{r}\right)^{1 / r}\right\|_{1} \\
& \leq 2^{1 / 2}\left(\|x\|_{1}^{r}+\|y\|_{1}^{r}\right)^{1 / r} \\
& \leq 2^{1 / r}\left(\|x\|_{1}^{2}+\|y\|_{1}^{2}\right)^{1 / 2}
\end{aligned}
$$

which implies $C_{\mathrm{NJ}}\left(\left(E,\|\cdot\|_{1}\right)\right) \leq 2^{2 / r-1}$. Consequently $\widetilde{C}_{\mathrm{NJ}}(E) \leq 2^{2 / p-1}$. This completes the proof.

13. Remark. In Theorem 10 the assertion (i) may be replaced by

$\left(\mathrm{i}^{\prime}\right) \widetilde{C}_{\mathrm{NJ}}(E)=2^{2 / p-1}$

if $l_{p}$ is finitely representable in $E$ or $E^{\prime}$ (cf. Remark 9).

By Theorem 10 we obtain the following result. 
14. Corollary. Let $E$ be a Banach lattice, and let $\widetilde{C}_{\mathrm{NJ}}(E)=2^{2 / p_{0}-1}, 1 \leq p_{0} \leq 2$. Then

$$
\begin{aligned}
p_{0} & =\sup \left\{p: E \text { is of type } p \text { and cotype } p^{\prime}\right\} \\
& =\sup \left\{p: E \text { and } E^{\prime} \text { are of type } p\right\} \\
& =\sup \left\{p: E \text { and } E^{\prime} \text { are of cotype } p^{\prime}\right\} \\
& =\sup \left\{p: E \text { is } p \text {-convex and } p^{\prime} \text {-concave }\right\} \\
& =\sup \left\{p: E \text { and } E^{\prime} \text { are } p \text {-convex }\right\} \\
& =\sup \left\{p: E \text { and } E^{\prime} \text { are } p^{\prime} \text {-concave }\right\} .
\end{aligned}
$$

The proof for the case $p_{0}=1$ goes in the same way as in the proof of Theorem 10 . (Note Lemma 1.f.3 of [13] for the last three identities.)

15. Remark. Let $E$ be a Banach lattice, and let $p(E), q(E)$ be as in Theorem D. Then the number $p_{0}$ in Corollary 14 is also represented as

$$
p_{0}=\min \left\{p(E), q(E)^{\prime}\right\}=2\left\{1+\log _{2} \widetilde{C}_{\mathrm{NJ}}(E)\right\}^{-1},
$$

where $1 / q(E)+1 / q(E)^{\prime}=1$.

\section{ACKNOWLEDGEMENT}

The authors thank the referee for some helpful comments.

\section{REFERENCES}

1. B. Beauzamy, Introduction to Banach spaces and their geometry, 2nd Ed., North Holland, Amsterdam-New York-Oxford, 1985. MR 88f:46021

2. J. A. Clarkson, Uniformly convex spaces, Trans. Amer. Math. Soc. 40 (1936), 396-414.

3. J. A. Clarkson, The von Neumann-Jordan constant for the Lebesgue space, Ann. of Math. $\mathbf{3 8}$ (1937), 114-115.

4. P. Enflo, Banach spaces which can be given an equivalent uniformly convex norm, Israel J. Math. 13 (1972), 281-288. MR 49:1073

5. E. Hewitt and K. Stromberg, Real and abstract analysis, Springer, New York-HeidelbergBerlin, 1965. MR 32:5826

6. R. C. James, Uniformly non-square Banach spaces, Ann. of Math. 80 (1964), 542-550. MR 30:4139

7. R. C. James, Super-reflexive Banach spaces, Canad. J. Math. 24 (1972), 896-904. MR 47:9248

8. P. Jordan and J. von Neumann, On inner products in linear metric spaces, Ann. of Math. 36 (1935), 719-723.

9. M. Kato and K. Miyazaki, Remark on generalized Clarkson's inequalities for extreme cases, Bull. Kyushu Inst. Tech., Math. Natur. Sci. 41 (1994), 27-31. MR 95h:46044

10. M.Kato and K. Miyazaki, On generalized Clarkson's inequalities for $L_{p}\left(L_{q}\right)$ and Sobolev spaces, Math. Japon. 43 (1996), 505-515. CMP 96:13

11. G. Köthe, Topologische lineare Räume I, Springer, Berlin-Heidelberg-New York, 1966. MR 33:3069

12. J. Kuelbs, Probability on Banach spaces, Marcel Dekker, New York-Basel, 1978. MR 80c: 60007

13. J. Lindenstrauss and L. Tzafriri, Classical Banach spaces II, Springer, Berlin-Heidelberg-New York, 1979. MR 81c:46001

14. B. Maurey and G. Pisier, Series de variables aleatoires vectorielles independantes et proprietes geometriques des espaces de Banach, Studia Math. 58 (1976), 45-90. MR 56:1388

15. G. Pisier, Martingales with values in uniformly convex spaces, Israel J. Math. 20 (1975), 326-350. MR 52:14940 
16. G. Pisier, Factorization of linear operators and geometry of Banach spaces, Amer. Math. Soc., Providence, RI, 1986. MR 88a:47020

17. G. Pisier and Q. Xu, Random series in the real interpolation spaces between the spaces $v_{p}$, Lecture Notes in Math., vol. 1267, Springer-Verlag, Berlin-Heidelberg-New York, 1987, pp. 185209. MR 89d:46011

Department of Mathematics, Kyushu Institute of Technology, Tobata, Kitakyushu 804, JAPAN

Department of System Engineering, Okayama Prefectural University, Soja 719-11, JAPAN 